\title{
A Retrospective Analysis of 303 Cases of Facial Bone Fracture: Socioeconomic Status and Injury Characteristics
}

\author{
Byeong Jun Kim, \\ Se ll Lee, \\ Chan Min Chung \\ Department of Plastic and Reconstructive \\ Surgery, National Medical Center, Seoul, \\ Korea
}

No potential conflict of interest relevant to this article was reported.

\begin{abstract}
Background: The incidence and etiology of facial bone fracture differ widely according to time and geographic setting. Because of this, prevention and management of facial bone fracture requires ongoing research. This study examines the relationship between socioeconomic status and the incidence of facial bone fractures in patients who had been admitted for facial bone fractures.

Methods: A retrospective study was performed for all patients admitted for facial bone fracture at the National Medical Center (Seoul, Korea) from 2010 to 2014. We sought correlations amongst age, gender, fracture type, injury mechanism, alcohol consumption, and type of medical insurance.

Results: Out of the 303 patients meeting inclusion criteria, 214 (70.6\%) patients were enrolled in National Health Insurance (NHI), 46 (15.2\%) patients had Medical Aid, and $43(14.2 \%)$ patients were homeless. The main causes of facial bone fractures were accidental trauma (51.4\%), physical altercation (23.1\%), and traffic accident (14.2\%). On Pearson's chi-square test, alcohol consumption was correlated significantly with accidental trauma $(p<0.05)$. And, the ratio of alcohol consumption leading to facial bone fractures differed significantly in the homeless group compared to the $\mathrm{NHI}$ group and the Medical Aid group $(p<0.05)$.

Conclusion: We found a significant inverse correlation between economic status and the incidence of facial bone fractures caused by alcohol consumption. Our findings indicate that more elaborate guidelines and prevention programs are needed for socioeconomically marginalized populations.
\end{abstract}

Keywords: Facial bone fracture / National Health Insurance / Homeless

\section{INTRODUCTION}

Because facial bone fractures represent common injuries, many studies have attempted to characterize these injuries. However, these characteristics differ across geographic settings (i.e., countries or regions within a country) as well as across time periods

\footnotetext{
Correspondence: Chan Min Chung

Department of Plastic and Reconstructive Surgery, National Medical Center, 245 Euliji-ro, Jung-gu, Seoul 04564, Korea

E-mail: nmcps.chung@gmail.com

Received October 1, 2015 / Revised October 22, 2015 / Accepted November 26, 2015
}

$[1,2]$. Accordingly, the incidence, type, and cause of recent cases of facial bone fracture must be studied in each new context, and such data is important in establishing prevention strategies and management guidelines for facial fractures.

In Korea, all citizens who have registered their residence with the government are entitled to coverage by the government-administered medical insurance system. Citizens are covered by either the National Health Insurance (NHI) program, within which medical expenses are partially covered by the government, or the Medical Aid program, within which most medical expenses are paid by the government for those of lower economic status 
(annual income less than $40 \%$ of the national median income). Only the homeless in the country are without any forms of health insurance because of the issues stemming from lack of fixed residence. This study examines the relationship between socioeconomic status and the incidence of facial bone fractures in $303 \mathrm{pa}-$ tients who had been admitted for facial bone fractures to the National Medical Center (Seoul, Korea).

\section{METHODS}

A single institutional retrospective study was conducted for patients who were diagnosed with facial fracture through physical examination and computed tomography (CT) scans from January 2010 to December 2014. Patient charts were reviewed for demographic information, fracture type, injury mechanism, alcohol consumption, and type of medical insurance.

To determine the percentage of each type of medical insurance of Korean citizens, the total number of people enrolled in any type of government-administered medical insurance, the total number of people eligible for $\mathrm{NHI}$, and the total number of people eligible for Medical Aid were identified. Similarly, specifically in Seoul, the number of people enrolled in any type of government-administered medical insurance, the number of people eligible for NHI, and the number of people eligible for Medical Aid provided by the NHI Service in 2014 were assessed. The total number of homeless recorded in the Investigation of the Current Status of Homeless in Korea conducted in 2012 were also collected as supplementary data, and their correlations were analyzed $[3,4]$.

(1) To minimize the selection bias, all patients were given a diagnosis of facial bone fracture at all departments including emergency medicine and dentistry as well as at department of plastic surgery, (2) The fracture had to have been identified in the CT conducted right after injury, (3) There could not be a past medical history of fracture in the area of the injury, and (4) To be able to compare to type of medical insurance of Seoul citizens, the patient's registered address had to be in the city of Seoul.

Fractures were classified as nasal, orbital, isolated zygomatic arch, zygomatic complex, nasoethmoidal orbital (NEO) complex, frontal, maxilla, mandible, or Le Fort fractures.
The cause of injury was classified as one of the following: traffic accidents, accidental trauma (e.g., tripping, falling, and/or running into an object or person), sports-related accidents, industrial accidents, physical altercation, and syncope. Traffic accidents were further differentiated into motorcycle, pedestrian, and automobile accidents.

Pearson's chi-square test, the $t$-test, and one-way analysis of variance (ANOVA) were for statistical evaluation using PASW ver. 18 (SPSS Inc., Chicago, IL, USA). A p-value less than 0.05 was considered as statistically significant.

\section{RESULTS}

The review identified 303 subjects ( 258 males and 45 females). The mean age was $42.0 \pm 16.5$ years (range, 8 to 87 years). In terms of age distribution, there were 2 subjects $(0.7 \%)$ less than 10 years of age, 28 subjects $(9.2 \%)$ aged $10-19,53$ subjects (17.4\%) in their twenties, 46 subjects (15.2\%) in their thirties, 71 subjects (23.4\%) in their forties, 63 subjects (20.8\%) in their fifties, 26 subjects (8.6\%) in their sixties, and 14 subjects (4.6\%) 70 years of age and above.

Of the 303 patients, health insurance coverage was provided by NHI for 214 patients (70.6\%) and by Medical Aid for 46 patients (15.2\%). The remaining 43 subjects (14.2\%) were homeless and did not have insurance coverage.

Insurance enrollment data and homeless population data for Seoul were obtained from respective government agencies. The population estimate for Seoul was 10,173,931 for 2014. Out of these, 9,949,301 residents (97.8\%) were enrolled in NHI program, and 224,630 (2.2\%) residents were enrolled in the Medical Aid program.

The homeless have no fixed residence and are not covered by health insurance programs. By 2012 estimates, South Korea had a population of 51,755,562 people. Out of these, 50,314,800 people (97.2\%) were covered by NHI and $1,440,772$ people (2.9\%) by Medical Aid. Homeless population accounted for 13,262 people $(50,314,800+1,440,772+13,262=51,768,824 ; 51,755,562)$.

The leading cause of injury was accidental trauma $(n=156$, $51.4 \%)$, followed by traffic accident $(43,14.2 \%)$, sports-related accident (19, 6.3\%), syncope (10,3.3\%), and industrial accident (5, 1.7\%) (Fig. 1). Of the 43 traffic accident injuries, motorcycle riders ac- 
counted for 17 cases (39.5\%), followed by motor vehicle passengers $(10,23.3 \%)$, pedestrians $(9,20.9 \%)$, and bicycle riders $(7,16.3 \%)$.

When cause of injury was classified according to gender, a high percentage of cases were males associated with all of the causes. When etiology was classified according to age groups, the most common etiologies in most age groups were accidental trauma, physical altercation, and traffic accidents, which comprised 80\%; since there were no industrial facilities nearby, the percentage of industrial accidents was below 3\% (Fig. 2).

When etiology was examined by the type of medical insurance, in the NHI group, there were 108 patients (50.5\%) of accidental trauma, 49 patients (22.9\%) of physical altercation, 27 patients (12.6\%) of traffic accidents, 19 patients (8.9\%) of sportsrelated accidents, 6 patients (2.8\%) of syncope, and 5 patients (2.3\%) of industrial accidents. In the Medical Aid group, there were 19 patients (41.3\%) of accidental trauma, 18 patients $(26.1 \%)$

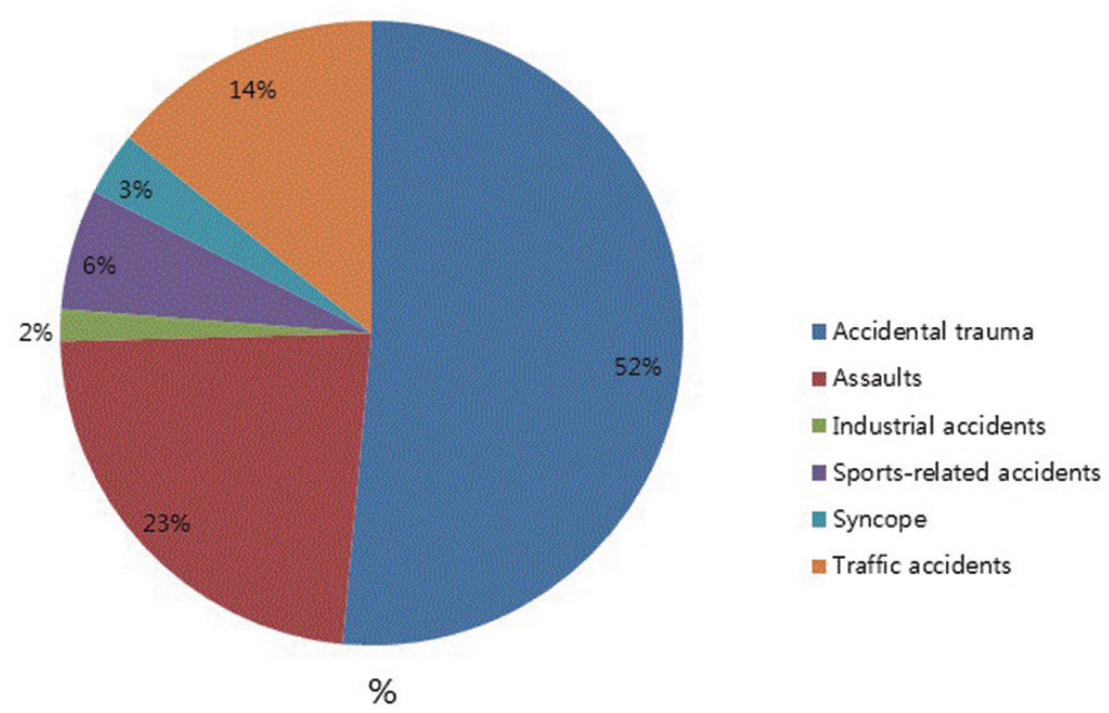

Fig. 1. Distribution of the fractures according to injury mechanism. More than half of the fractures (156; 51.4\%) were caused by accidental trauma, followed by physical altercation and traffic accidents.

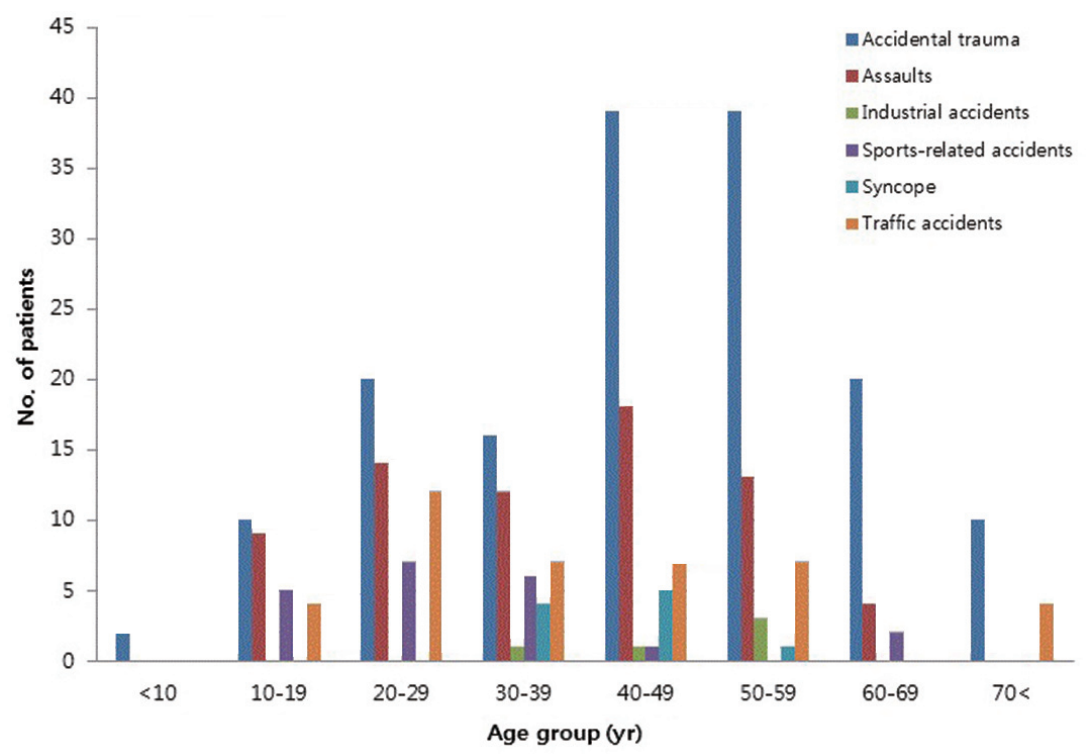

Fig. 2. Injury mechanism by age group. In most age groups, the most common injury mechanisms were accidental trauma, physical altercation, and traffic accidents. The percentage of sports-related accidents drastically declined after 40 years of age. 
of physical altercation, 11 patients (23.9\%) of traffic accidents, 4 patients $(8.7 \%)$ of syncope, and in the homeless group, there were 43 patients $(67.4 \%)$ of accidental trauma, 9 patients $(20.9 \%)$ of physical altercation, and 5 patients (11.6\%) of traffic accidents (Fig. 3). When the etiology of facial bone fracture was compared among the groups with different types of medical insurance, there was no significant difference among the groups in terms of the proportion of accidental trauma, physical altercation, or traffic accidents, which together accounted for the etiology of the majority of cases in every group (one-way ANOVA, $p>0.05$ ).

When the direct and indirect association between etiology and alcohol consumption was examined, among the 104 patients (34.3\%) involving alcohol consumption, 73 patients (46.8\%) were caused by accidental trauma, 24 patients $(34.3 \%)$ by physical altercation, and 7 patients (16.3\%) by traffic accidents, with no patients of sports-related accidents, syncope, or industrial accidents. Alcohol consumption was significantly correlated with accidental trauma (Pearson's chi-square test, $p<0.05$ ); however, alcohol consumption showed no significant difference from both physical altercation and traffic accidents (Pearson's chi-square test, $p>0.05$ ) (Table 1 ).

When the correlation between the incidence of facial bone fracture according to the type of medical insurance and alcohol consumption was examined, 65 patients involving alcohol (30.4\%) were found to have NHI, 11 patients (23.9\%) had Medical Aid, and 28 patients $(65.1 \%)$ were homeless. The ratio of fractures involving alcohol consumption did not differ significantly between the NHI group and the Medical Aid group (Pearson's chi-square test, $p>0.05$ ), while it did differ significantly in the homeless group compared to the ratio of alcohol consumption in the NHI group and the Medical Aid group (Pearson's chi-square test, $p<0.05$ ) (Table 2).

When the fracture types were examined, there were 134 cases (41\%) of nasal fractures, 70 cases $(21.4 \%)$ of zygomatic complex fractures, 60 cases (18.3\%) of orbit fractures, 31 cases (9.5\%) of

Table 1. Association of alcohol-related facial fracture with etiologies

\begin{tabular}{|lrrrcc} 
Causes & Alcohol & No alcohol & $p$-value & adds ratio & Od \\
Accidental trauma & 73 & 83 & $<0.001$ & 3.291 \\
\hline Non-accidental trauma & 31 & 116 & & 1.000 \\
\hline Physical altercation & 24 & 46 & 0.994 & 0.998 \\
\hline Non-physical altercation & 80 & 153 & & 1.000 \\
\hline Traffic accidents & 7 & 36 & 0.007 & 0.327 \\
\hline Non-traffic accidents & 97 & 163 & & 1.000 \\
\hline
\end{tabular}

Values are presented as the number of patients.

Evaluation showed a statistically significant association of alcohol-related facial fracture with accidental trauma.

a) Pearson's chi-square test.

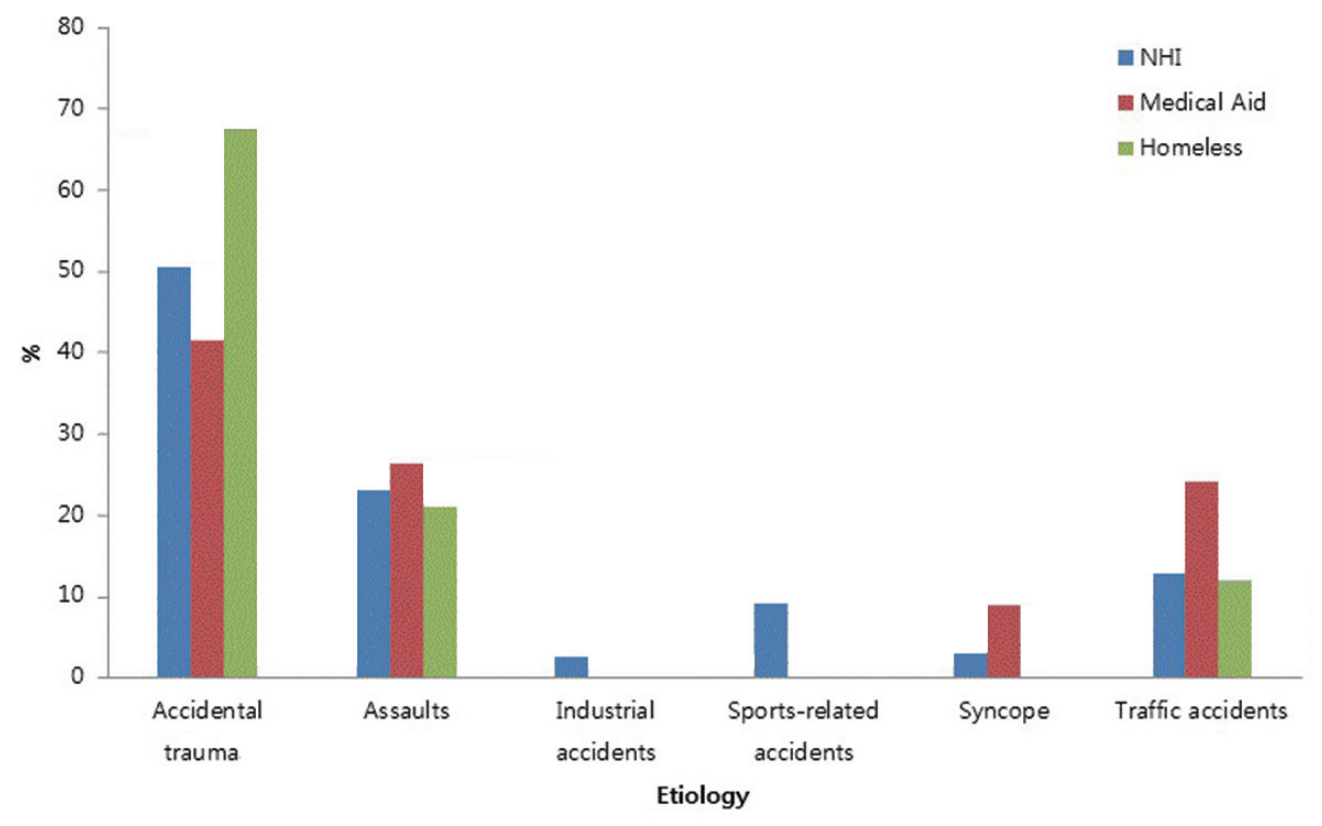

Fig. 3. Medical insurance type and injury mechanism. For all types of health insurance coverage, the most common injury mechanisms were accidental trauma, physical altercation, and traffic accidents. NHI, National Health Insurance. 
mandible fractures, 12 cases (3.7\%) of isolated zygomatic arch fractures, 9 cases (2.6\%) of NEO complex fractures, and 11 cases (3.4\%) of other fractures (Fig. 4). Among the total patients, 23 cases (7.6\%) presented with fractures in 2 or more different areas.

When the association between the fracture types and alcohol consumption was examined, there were 46 cases $(41.8 \%)$ of nasal fractures, 25 cases (22.7\%) of zygomatic complex fractures, 21 cases $(18.1 \%)$ of orbit fractures, 8 cases $(7.8 \%)$ of mandible fractures. The percentage of fracture types showed no significant difference between groups associated with alcohol and non-alcohol.

When the fracture types was examined by the type of medical insurance, in the NHI group, there were 104 cases (48.6\%) of nasal fractures, 42 cases (19.6\%) of zygomatic complex fractures, 39 cas-

Table 2. Association of alcohol-related fracture with the type of medical insurance

\begin{tabular}{lrrrrc}
\hline Type of medical insurance & Alcohol & No alcohol & $p$-value & Odds ratio & Od \\
\hline National Health Insurance & 65 & 149 & 0.382 & 0.720 \\
\hline Medical aid & 11 & 35 & & 1.000 \\
\hline National Health Insurance & 65 & 149 & $<0.001$ & 1.000 \\
\hline Homeless & 7 & 36 & & 18.263 \\
\hline Medical aid & 11 & 35 & $<0.001$ & 1.000 \\
\hline Homeless & 7 & 36 & & 5.952 \\
\hline
\end{tabular}

Values are presented as the number of patients.

Evaluation showed a statistically significant association between the homeless group and alcohol consumption.

${ }^{a}$ Pearson's chi-square test. es $(18.2 \%)$ of orbit fractures, 19 cases $(8.8 \%)$ of mandible fractures, in the Medical Aid group, there were 17 cases (37.0\%) of nasal fractures, 16 cases (34.8\%) of zygomatic complex fractures, 6 cases (13.0\%) of orbit fractures, 6 cases (13.0\%) of mandible fractures, in the homeless group, there were 13 cases (30.2\%) of nasal fractures, 12 cases $(27.9 \%)$ of zygomatic complex fractures, 6 cases (14.0\%) of mandible fractures, 5 cases (11.6\%) of orbit fractures. When the type of facial bone fracture was compared among the groups with different types of medical insurance, the percentage of zygomatic complex fracture and mandible fractures in the Medical Aid group and the homeless group was relatively very high compared to the percentage in the NHI group.

\section{DISCUSSION}

In South Korea, the incidence of trauma has been increasing because of the diversification of industries, increasing elderly population, increasing number of traffic accidents, and promotion of leisurely physical activity. With this increase in the general incidence of trauma, concomitant increase has been observed for facial bone fractures. Facial bone fracture patients frequently present to plastic surgeons, and a general understanding of the injury pattern is crucial in managing these fractures and restoring the aesthetics and function of the facial bones. Many studies have investigated the in-
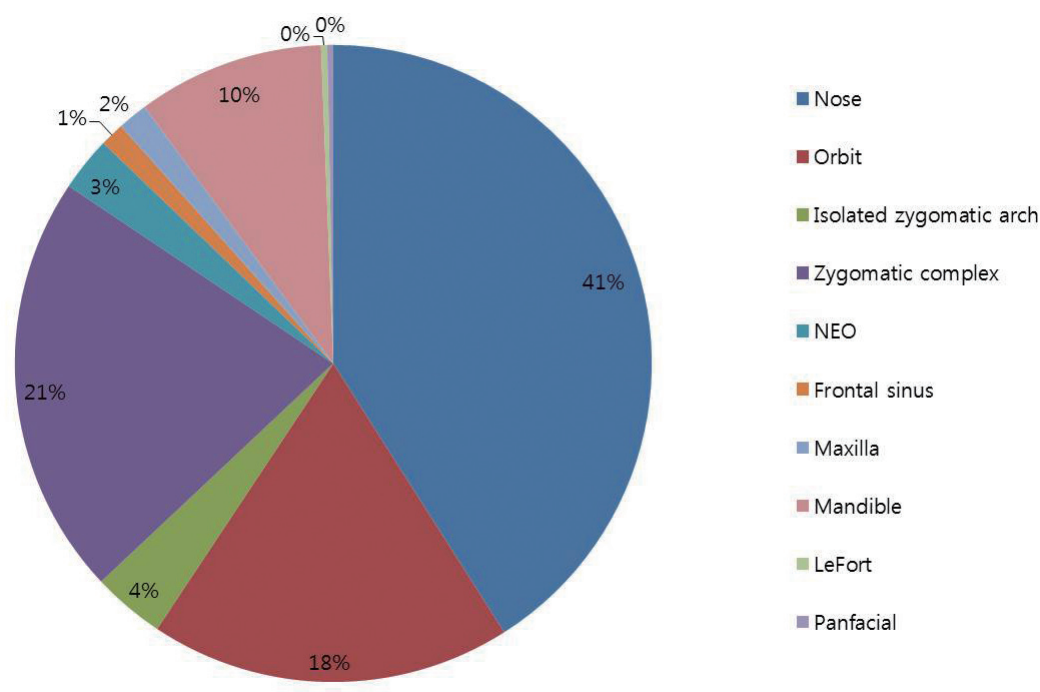

Fig. 4. Distribution of the fractures according to fracture type. Nasal fractures, zygomatic complex fractures, and orbit fractures comprised more than $80 \%$ of all facial bone fractures. NEO, nasoethmoidal orbital. 
cidence and etiology of facial bone fractures. However, in Korea, few studies have investigated the potential correlation between the socioeconomic status and facial fracture patterns. In South Korea, greater than $90 \%$ of the population is enrolled in the NHI program. Therefore, given that this study distinguished the Medical Aid group and the homeless group, who account for less than $10 \%$ of Korean citizens, from the rest of the population, it can be said to have compared the characteristics of facial fracture in a low socioeconomic group with those of the remaining group.

In our review, the majority of facial fractures could be attributed to three type of injury mechanisms: accidental trauma, physical altercation, and traffic accident. In a recent study conducted in Korea, physical altercation was reported to be the most common cause of facial fracture $[5,6]$, whereas in this study, accidental trauma was found to be the most common etiology. This could be attributed to selection bias, but could also be due to the expansion of the definition of 'accidental injury' in Korea, which now includes not just simple falls but also physical impact from running or slipping into solid objects. In older epidemiologic studies conducted in Korea, traffic accidents had been reported to be the most common injury mechanism behind facial fractures $[7,8]$. However, more recent studies have shown that facial bone fractures due to traffic accidents has decreased significantly $[5,6,9]$. This shift in injury pattern could be explained by increased seatbelt wearing, mandatory regulations for airbags, and improvements in the traffic environment [10].

Many studies have reported alcohol consumption to be associated with accidental trauma and physical altercation. Johnston and McGovern [11] have reported that, as blood alcohol concentration increased, the frequency and the severity of facial injury caused by falling accidents increased as well. The authors proposed the inhibition of protective reflexes due to alcohol consumption as the reason behind this observation, which is a finding we also observe in our patient population. In addition, Lee [12] have found that alcohol consumption is a critical factor contributing to physical altercation [13]. This is a finding we did not observe in our study. While alcohol consumption may increase the incidence of physical altercation, our results suggest that alcohol consumption does not directly cause facial bone fracture through physical altercation. Additionally, there are many errors in retrospective studies related to alcohol consumption because patients often try to hide their drinking history or because patients are often hospitalized several days after the injury. As a result, data regarding drinking history is not always accurate in retrospective analysis. When the type of medical insurance was examined, alcohol-related facial bone fractures was higher for the homeless population compared to either the NHI or the Medical Aid patients.

Among studies on the relationship between trauma and economic status of patients, Mosenthal et al. [14] have reported that, excluding work-related falling incidents, $52 \%$ of patients with fall injuries were unemployed at the time of investigation. Furthermore, a majority of fall patients who are unemployed (83\%) had alcohol or drug addiction, which suggests that individual economic status is correlated with risk for falling injury. Similarly, we have found that the type of health insurance had significant patterns with respect to the incidence of facial bone fracture associated with alcohol consumption. In other words, we identified a significant inverse correlation between economic status and the incidence of facial bone fractures caused by alcohol consumption. This association was accentuated in the homeless group because homeless people are vulnerable to living in insecure settings, the possibility of developing medical problems is greater. Moreover, homeless patients are at greater risk of psychological problems associated with socioeconomic circumstances and, accordingly, a greater possibility of relying on alcohol, which may result in a vicious cycle of injury and injury promoting behavior. For patients of low-socioeconomic status, such cycle can increase the difficulty of returning as active participants within a society. From a wider perspective, this phenomenon places increasing burdens on the society through greater health care expenditure. Consequently, government agencies and health professionals should attempt to store and analyze such data and establish more elaborate guidelines and prevention programs for the socially marginalized patients.

While many clinico-epidemiological studies on facial bone fracture have been published, the majority of these studies have only analyzed the incidence of facial bone fracture within a given region or treated at a single institution. Furthermore, the studies have not examined the economic status of each individual patient. Therefore, the present study is significant in that it has analyzed 
the relationship between the socioeconomic status of patients to the characteristics of facial bone fracture.

\section{REFERENCES}

1. van Hoof RF, Merkx CA, Stekelenburg EC. The different patterns of fractures of the facial skeleton in four European countries. Int J Oral Surg 1977;6:3-11.

2. Haug RH, Foss J. Maxillofacial injuries in the pediatric patient. Oral Surg Oral Med Oral Pathol Oral Radiol Endod 2000;90:126-34.

3. The National Health Insurance Corporation. National Health Insurance service yearbook 2014. Seoul: The National Health Insurance Corporation; 2014.

4. The Ministry of Health and Welfare. Investigation of the conditions of disadvantaged households in Korea. Sejong: Ministry of Health and Welfare; 2011.

5. Yang EZ, Kim CY. The relationship between socioeconomical status and incidence of facial bone fracture. J Korean Soc Plast Reconstr Surg 2011;38:263-72.
6. Hwang K, You SH. Analysis of facial bone fractures: an 11-year study of 2,094 patients. Indian J Plast Surg 2010;43:42-8.

7. Kwon HJ, Han J, Kim JH, Jung HY, Kim JY, Yoon SH, et al. Clinical epidemiologic study of facial bone fractures in Daegu. J Korean Soc Plast Reconstr Surg 2007;34:365-70.

8. Kang MS, Choi BC, Kim YH, Woo SH, Jeong JH, Seul JH. An analysis of 1,210 facial bone fractures in 835 patients: 5 year survey. J Korean Soc Plast Reconstr Surg 1998;25:598-606.

9. Suh YH, Kim YJ. Statistical analysis of factors associated with facial bone fractures. Arch Craniofac Surg 2012;13:36-40.

10. Davis JW, Bennink L, Kaups KL, Parks SN. Motor vehicle restraints: primary versus secondary enforcement and ethnicity. J Trauma 2002;52:225-8.

11. Johnston JJ, McGovern SJ. Alcohol related falls: an interesting pattern of injuries. Emerg Med J 2004;21:185-8.

12. Lee KH. Interpersonal violence and facial fractures. J Oral Maxillofac Surg 2009;67:1878-83.

13. Borges G, Cherpitel CJ, Rosovsky H. Male drinking and violence-related injury in the emergency room. Addiction 1998;93:103-12.

14. Mosenthal AC, Livingston DH, Elcavage J, Merritt S, Stucker S. Falls: epidemiology and strategies for prevention. J Trauma 1995;38:753-6. 